\title{
Enseñanza remota durante la pandemia por COVID-19 en la Universidad Tecnológica del Norte de Aguascalientes
}

\section{Remote teaching during the COVID-19 pandemic at the Universidad Tecnológica del Norte de Aguascalientes}

\author{
ÁLVAREZ-LOERA, Ana Cecilia†** \& PÉREZ-MARTÍNEZ, María Guadalupe \\ Universidad Tecnológica del Norte de Aguascalientes, Av. Universidad No. 10001. Estación Rincón, Rincón de Romos, Ags., \\ C.P. 20400, México. \\ ID $1^{\mathrm{er}}$ Autor: Ana Cecilia, Álvarez-Loera / ORC ID: 0000-0002-6506-5310, CVU CONACYT ID: 208972 \\ ID $1^{\mathrm{er}}$ Coautor: María-Guadalupe, Pérez-Martínez / ORC ID: 0000-0003-3655-0090, CVU CONACYT ID: 38222
}

\begin{abstract}
Resumen
Este artículo expone un estudio exploratorio de las experiencias de docentes universitarios durante la Enseñanza Remota de Emergencia (ERE) en respuesta a la COVID-19. Desde un enfoque sistémico, se indagaron las prácticas de enseñanza, las condiciones para la enseñanza remota y las condiciones laborales de los profesores. Se aplicó una encuesta a todos los docentes de la Universidad Tecnológica del Norte de Aguascalientes a través de internet. Se identificó sobrecarga de actividades académicas entre los docentes, incremento de la jornada laboral por actividades de planificación y evaluación de clases en ambientes virtuales y distintas fuentes de estrés entre los profesores. Los hallazgos muestran algunas dificultades enfrentadas por la institución y enfatizan la importancia de cuidar la provisión de insumos que contribuyan a mejorar los ambientes para la enseñanza y el aprendizaje, preservar la salud de los docentes y estudiar la influencia que tienen las condiciones estudiadas en las prácticas docentes.
\end{abstract}

Educación remota, Educación superior, Pandemia

\begin{abstract}
This article presents an exploratory study of the experiences of university teachers during Emergency Remote Teaching (ERE) in response to COVID-19. Teaching practices, conditions for remote teaching and teachers' working conditions were investigated under a systematic approach. An internet survey was applied to all teachers at the Universidad Tecnológica del Norte de Aguascalientes. Results show an overload of academic activities among teachers, an increase in working hours due to planning and assessment in online environments, and other sources of stress among teachers. Some difficulties faced by the institution are hightlighted and conclusions emphasize the importance of ensuring inputs that contribute to improving teaching and learning environments, preserving the health of teachers and continuing the research on the influence of different teaching conditions on teaching practices.
\end{abstract}

Remote education, Higher education, Pandemics

Citación: ÁLVAREZ-LOERA, Ana Cecilia \& PÉREZ-MARTínEZ, María Guadalupe. Enseñanza remota durante la pandemia por COVID-19 en la Universidad Tecnológica del Norte de Aguascalientes. Revista de Políticas Universitarias. 2020. 4-12:11-19.

\footnotetext{
* Correspondencia al Autor (Correo electrónico: ana.alvarez@utna.edu.mx)

$\dagger$ Investigador contribuyendo como primer autor.
} 


\section{Introducción}

En el mes de marzo de 2020, la Organización Mundial de la Salud declaró la emergencia sanitaria mundial ante la pandemia por la enfermedad por COVID-19. En México, las autoridades educativas determinaron la suspensión de actividades presenciales en todos los niveles del sistema educativo nacional, como medida preventiva para disminuir el impacto en la salud de la población.

Se determinó una primera suspensión durante el mes de abril y la Secretaría de Educación Pública emitió algunos lineamientos para las instituciones públicas de educación superior. Entre ellas, la impartición de clases por medios digitales a distancia y de comunicación con los estudiantes, así como ajustes a los calendarios escolares y programas educativos (Diario Oficial de la Federación, 16 de marzo de 2020; Secretaría de Educación Pública, 2020)

Se estableció también que la medida de suspensión aplicaría solo a los estudiantes; el personal académico y administrativo continuaría ofreciendo servicios a través de medios electrónicos. Dicha decisión se ha ido ampliando debido a que no ha cesado la contingencia sanitaria. Estimando los efectos perjudiciales que podría tener el cambio de actividades presenciales a remotas, se ha valorado que las instituciones tendrían que transitar hacia modelos híbridos en educación (Organización Mundial de la Salud, 2020).

En la Universidad Tecnológica del Norte de Aguascalientes (UTNA) se imparten programas de técnico superior universitario y licenciatura, los cuales tienen la característica de ser cuatrimestrales. La primera suspensión de actividades ocurrió a finales del cuatrimestre enero-abril de 2020, por lo que se migró de manera inmediata de la enseñanza presencial a la enseñanza remota. En ese momento la institución no disponía de una plataforma virtual, por lo que, los docentes hicieron uso de diversas herramientas de comunicación y enseñanza a distancia con los estudiantes. Los docentes trabajaron en algunos periodos dentro de la institución y en otros momentos desde sus hogares.
Para dar inicio al cuatrimestre mayoagosto de 2020, se desarrolló el Aula Virtual UTNA, que es una plataforma Moodle, para instrumentar la educación remota.

Como una estrategia para fortalecer la práctica docente se impartió el curso Entornos Virtuales de Aprendizaje, con el fin de apoyar el uso de la plataforma virtual y desarrollar en los profesores habilidades en el manejo de herramientas de enseñanza-aprendizaje por competencias en entornos de educación virtual y a distancia. Se ofreció a los académicos una guía con lineamientos y sugerencias para la gestión de las clases. En este contexto de cambio educativo de emergencia, se consideró necesario explorar cómo se estaban instrumentando las clases a distancia, así como conocer las condiciones institucionales, de los docentes y de los estudiantes para enfrentar esta nueva condición.

El estudio tuvo el propósito de explorar las prácticas y condiciones para la enseñanza y el aprendizaje durante el periodo de enseñanza remota instrumentada ante la pandemia por COVID-19, en la Universidad Tecnológica del Norte de Aguascalientes. En el primer apartado se presenta el marco teórico; en el segundo se describe la metodología empleada; en el tercero se muestran los resultados obtenidos $y$ finalmente se apuntan algunas conclusiones.

\section{Marco teórico}

La emergencia sanitaria por la COVID-19 llevó a la suspensión de interacciones presenciales en el sector educativo. La mayoría de la población estudiantil mundial, considerando todos los niveles y modalidades, se vio afectada por el cierre de escuelas, lo que ha hecho que se catalogue esta contingencia como la interrupción educativa más prolongada (United Nations, 2020, p. 5), y de la cual se anticipan consecuencias relacionadas con la ampliación de brechas de aprendizaje.

En Educación Superior, el cierre de escuelas ha afectado a cerca de 220 millones de estudiantes a nivel mundial, y en Latinoamérica esta cifra asciende a más de 27 millones de estudiantes (World Bank, 2020, p. 1). 
Ante este fenómeno de amplias dimensiones, las instituciones de educación superior debieron instrumentar medidas remotas para continuar la provisión de servicios educativos resguardando la seguridad de la comunidad escolar.

Las medidas educativas implementadas durante la contingencia sanitaria se han denominado en la literatura como Enseñanza Remota de Emergencia (ERE) (Bozkurt \& Sharma, 2020; Casillas \& Rodríguez, 2020; Galindo et al., 2020; Hodges et al., 2020; Whittle et al., 2020). Este término se ha acuñado para distinguir el cambio temporal en la modalidad de prestación de servicios educativos en respuesta a una crisis que impide que se brinden como habían sido planeados; este cambio es temporal pues se espera volver a la modalidad habitual en cuanto haya terminado la contingencia.

Una de las herramientas empleadas en la ERE ha sido el internet (Rugube et al., 2020), a través del cual los docentes y estudiantes acceden a materiales y sostienen interacciones. También se han documentado experiencias de instituciones de educación superior en las que emplean medios tales como radio, televisión, correo postal, entre otros (World Bank, 2020). Las decisiones sobre los medios para suministrar servicios educativos han sido influenciadas por las características de las instituciones de educación superior, de los docentes y de la población estudiantil que atienden.

La provisión de la ERE a través de internet requiere, por ejemplo, que las instituciones cuenten con equipamiento tecnológico, plataformas de gestión de aprendizaje y conectividad específicas; además, los docentes y los estudiantes también requieren infraestructura física y tecnológica en sus hogares. Aunque las instituciones debieron realizar evaluaciones rápidas de este tipo de condiciones, se anticipa que los retos en la provisión de ERE son múltiples (World Bank, 2020).

Aunque el internet ha sido uno de los medios para continuar la provisión de servicios educativos durante la emergencia sanitaria, los especialistas han hecho un llamado para distinguir este tipo de enseñanza con respecto de la educación en línea (Hodges et al., 2020).
Pues la educación en línea usa el internet para promover la interacción entre el docente, los estudiantes y el contenido, y además, proveer apoyo durante el proceso de aprendizaje (Ally, 2008), y esto se hace a través de un diseño instruccional planificado específicamente para proveer la educación en este ambiente de aprendizaje.

Se ha encontrado que la efectividad de este tipo de enseñanza recae en un diseño instruccional cuidadoso con base en modelos sistemáticos (Means et al., 2014), la preparación de los docentes y el apoyo institucional que se les provea para desarrollar sus funciones, la construcción de un sentido de comunidad de aprendizaje en línea, y, las interacciones que se promuevan entre docentes, contenido y estudiantes (Sun \& Chen, 2016). Por otro lado, el diseño instruccional que se realice dependerá del tamaño de los grupos y el personal docente a cargo de ellos; la cantidad de alumnos en cada grupo determinará el tipo de actividades que llevarán a cabo los estudiantes además de la retroalimentación que brinden los docentes. De hecho, algunos autores señalan que el diseño de un curso en línea requiere de seis a nueve meses de trabajo previo a su implementación (Hodges et al., 2020).

Dada la velocidad con la cual las instituciones de educación superior tuvieron que migrar la enseñanza presencial a otros medios, en especial a través de internet, es probable que no hayan contado con las condiciones mencionadas, en específico: tiempo para rediseñar los cursos de acuerdo con la nueva modalidad de impartición, modelos específicos para diseño instruccional, apoyo profesional para diseñar los cursos, capacidad para modificar condiciones tales como tamaño de los grupos o personal docente a cargo de la enseñanza de cada materia por grupo.

Bajo las condiciones reales que tuvieron las instituciones de educación superior para la transición durante la emergencia sanitaria es probable las respuestas diseñadas por el cuerpo docente hayan sido rápidas y sean una fuente de estrés; de la misma manera, la implementación de educación a través de internet bajo condiciones inadecuadas puede llevar a un "distanciamiento pedagógico entre los estudiantes y los docentes y el proceso de aprendizaje" (Rugube et al., 2020, p. 120). 
Lo anterior muestra la necesidad de aproximarse al estudio de los fenómenos educativos durante la ERE considerando el contexto que enfrentaron la instituciones, los insumos con los que contaron, los procesos que implementaron, y los resultados que se han logrado (Stufflebeam \& Zhang, 2017). Parece conveniente indagar sobre la infraestructura tecnológica y el personal de apoyo para la docencia con la que contaban las instituciones para la transición, los recursos que se proveyeron para la migrar la modalidad de enseñanza, el tipo de actividades que implementaron los docentes durante la contingencia y las interacciones que promovieron con los estudiantes, las dificultades que enfrentaron los docentes, y los resultados que se alcanzaron en este periodo de tiempo.

Este tipo de investigaciones son indispensables debido a la incertidumbre en relación con la duración de la emergencia sanitaria, que hace suponer que se seguirán proveyendo servicios educativos de forma remota, por lo que las instituciones requieren elementos para enriquecer las experiencias de diseño de programas y su implementación.

\section{Metodología}

El estudio es exploratorio, por tratarse de un fenómeno de investigación sin precedente, y tiene un diseño tipo encuesta, en el que se miden "comportamientos, pensamientos o condiciones objetivas de la existencia de los participantes en una investigación a fin de establecer una o varias relaciones de asociación entre un fenómeno y sus determinantes" (Giroux \& Tremblay, 2004, p. 98).

La información se recolectó a través de un cuestionario aplicado a través de la herramienta de Google Formularios. El cuestionario comprendía los siguientes temas: procesos de la práctica docente remota en su fase interactiva, tales como: gestión de la clase, evaluación de los aprendizajes y comunicación con los estudiantes; insumos, denominados condiciones para la enseñanza remota, entre ellos, capacitación recibida, la disponibilidad de equipo de cómputo y conexión a internet; además, situaciones enfrentadas por los docentes durante la pandemia; y, algunas condiciones laborales de los profesores.
El vínculo de internet para responder cuestionario se envió a todos los profesores que impartieron clases durante el cuatrimestre mayoagosto de 2020. El periodo para dar respuesta al cuestionario fue del 23 de julio al 7 de agosto de 2020 .

La población total de académicos en la UTNA es de 158 profesores. Se promovió la participación voluntaria y se obtuvo una tasa de respuesta del 79\% (125 docentes). Los docentes participantes pertenecen a las siguientes áreas: 24\% a la Dirección Académica de Tecnología de la Información y Mecatrónica, $17 \%$ a la Dirección Académica de Ingenierías, 21\% a la Dirección Académica de Desarrollo de Negocios y Agricultura, 19\% a la Dirección Académica de Administración y Contaduría, $17 \%$ al Departamento de Idiomas y Comunicación y $2 \%$ a la Subdirección de Atención al Cliente.

La información se analizó haciendo uso de estadística descriptiva, con el software estadístico SPSS. Los resultados se presentaron ante el Consejo Académico de la UTNA en el mes de agosto, con la finalidad de apoyar la toma de decisiones para instrumentar el último cuatrimestre agosto-diciembre 2020.

\section{Resultados}

Los resultados se organizan en tres partes: a) condiciones laborales de los docentes; b) prácticas docentes en la educación remota; y, c) condiciones para el trabajo docente remoto.

Con respecto las condiciones laborales de los docentes, se indagó el tipo de contratación. Lo resultados muestran que la mayoría de los participantes son profesores de asignatura (PA) $(70 \%)$, y el resto son profesores de tiempo completo (PTC).

Los PTC son el personal académico de la universidad contratado para realizar funciones sustantivas de docencia, investigación, vinculación, gestión administrativa y actividades orientadas a extender los beneficios de la ciencia, la técnica y la cultura en los términos de las disposiciones que al respecto se expidan y de los planes y programas académicos que se aprueben. Los profesores de asignatura (PA) son el personal académico contratado por tiempo determinado por la universidad para el desarrollo de sus funciones sustantivas de docencia $y$ vinculación. 
Se exploraron las actividades académicas que realizan los docentes, tales como: impartir clases, asesorar estadías, brindar asesoría académica, tutoría, comisiones académicas y brindar asesoría psicopedagógica. Como se puede apreciar en el gráfico 1, las actividades de impartir clases como asesorar estadías se concentra en los profesores de asignatura; mientras que la asesoría académica, tutorías y otras comisiones académicas en los profesores de tiempo completo. $\mathrm{La}$ asesoría psicopedagógica está a cargo de dos profesionales asignados a esta función. Todos los profesores de tiempo completo reportaron llevar a cabo otras actividades, además de las indagadas.

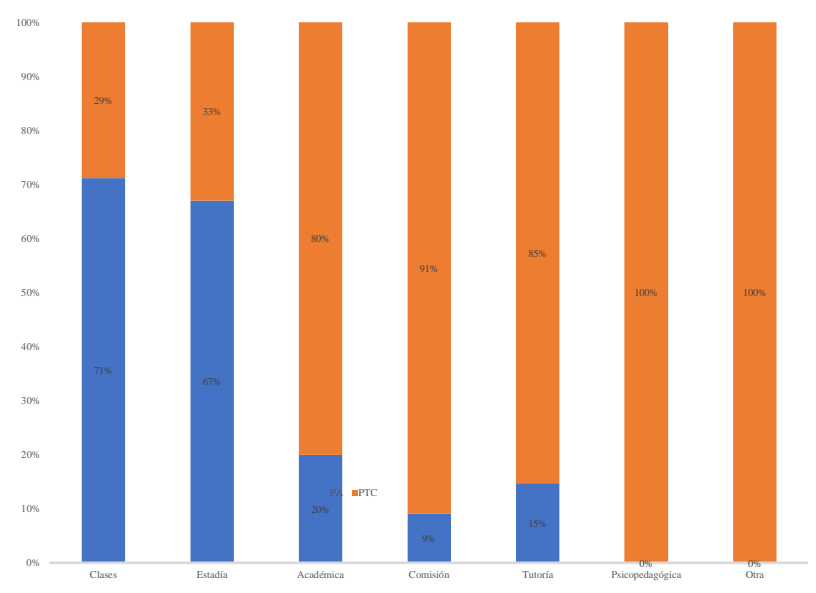

Gráfico 1 Actividades académicas que realizan los docentes

Los profesores atienden en promedio 4 grupos de estudiantes, aproximadamente 70 alumnos por docente. La información es similar por área académica.

En cuanto a la dedicación de horas frente a grupo, se encontró que $46 \%$ de los docentes tiene una dedicación de hasta 10 horas, $37 \%$ hasta 20 horas, $15 \%$ hasta 30 horas y $2 \%$ hasta 40 horas. La distribución media de horas frente a grupo es similar entre docentes de asignatura y de tiempo completo. Por otro lado, $65 \%$ de los docentes participantes tiene dedicación exclusiva a la UTNA, el resto trabaja en otra institución educativa impartiendo clases; de ellos, el $13 \%$ reportó una dedicación de hasta 10 horas, $20 \%$ hasta 20 horas y $1 \%$ más de 20 horas.

A fin de instrumentar la educación remota, la coordinación de capacitación docente emitió los lineamientos iniciales para el uso del aula virtual UTNA.
Estos incluyen recomendaciones y sugerencias acerca del manejo de la plataforma, desarrollo de la dinámica de clase y precisiones sobre la atención de condiciones específicas de alumnos y grupos, por consiguiente, constituyen un referente inmediato para estudiar las prácticas docentes.

Con respecto a las prácticas docentes durante el periodo indagado, se preguntó acerca de las herramientas tecnológicas que utilizaron en sus clases. El gráfico 2 muestra que todos los profesores utilizan el aula virtual UTNA como principal herramienta para sus impartir clases y que la complementan con otras herramientas: $79 \%$ utiliza plataformas de aulas virtuales tales como Classroom, Edmodo, Blackboard, entre otros; $85 \%$ utiliza herramientas para videoconferencias como Google Meet, Zoom o Skype. Se emplean otros medios de comunicación como correo electrónico (91\%), WhatsApp (90\%), llamadas telefónicas (52\%), y repositorios de archivo, como Google Drive, One Drive, Dropbox (48\%). Las redes sociales como Facebook, Instagram o Twitter son menos utilizadas (16\%).

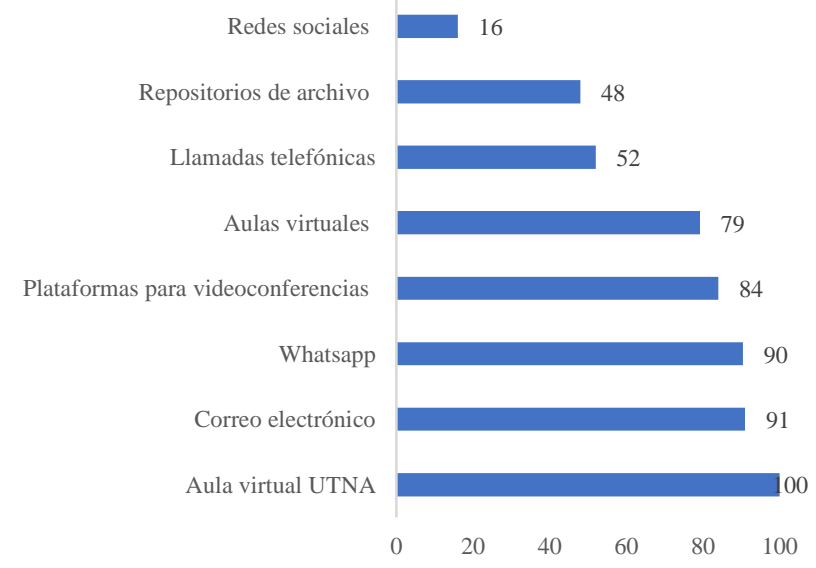

Gráfico 2 Herramientas tecnológicas empleadas por los docentes

Se indagó la frecuencia con que los docentes realizan videoconferencias con sus estudiantes; en la institución se ha recomendado que se realice esta actividad, aunque no se ha sugerido una frecuencia específica. Se encontró que la mayoría de los docentes emplea videoconferencias en sus clases, con diferentes frecuencias: $22 \%$ basa sus clases en este medio de comunicación, pues lo emplea en todas las sesiones, $24 \%$ al menos una vez por semana y $46 \%$ solamente algunas clases; $7 \%$ de los profesores no realiza esta actividad. 
Se exploró el tiempo que destinan los docentes a actividades de planeación y evaluación, por cada hora de clase. Como se puede apreciar en el gráfico 3, el tiempo que se destina tanto a la planeación como a la evaluación es similar. Con respecto a la planeación, una proporción mínima destina menos de una hora, $45 \%$ dedica de una a dos horas, $46 \%$ tres horas o más. Con respecto a la evaluación, $1 \%$ de los docentes refiere dedicar menos de una hora, $42 \%$ de una a dos horas, y $46 \%$ tres horas o más.

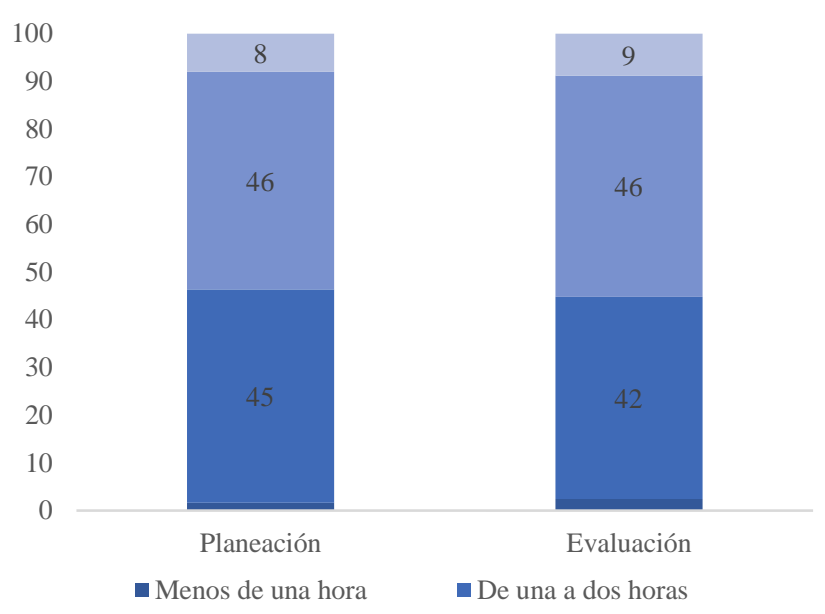

Gráfico 3 Docentes según tiempo destinado a planeación y evaluación de clases

Se encontró también que la mayoría de los docentes percibe que está destinando más tiempo a actividades de planeación y evaluación que el que dedicaban antes de la emergencia sanitaria $(80 \%)$.

La enseñanza remota introdujo cambios en la organización de las clases y los estudiantes tuvieron que ajustar sus maneras de aprender, esto ha modificado la interacción entre profesores y estudiantes, lo cual no ha estado exento de algunos problemas. Los docentes reportan algunas dificultades académicas relacionadas con la enseñanza y el aprendizaje durante el trabajo a distancia y desde casa. En el gráfico 4 se muestra que $80 \%$ ha presentado fallas en su servicio de internet, seis de cada diez han tenido problemas con la velocidad del servicio, fallas en el aula virtual y dificultades de conexión de parte de los estudiantes. Reporta también la mitad de los docentes su apreciación acerca del desinterés de los alumnos, muchos de ellos entregan trabajos fuera de tiempo, lo cual ha contribuido a incrementar el número de horas de trabajo docente.

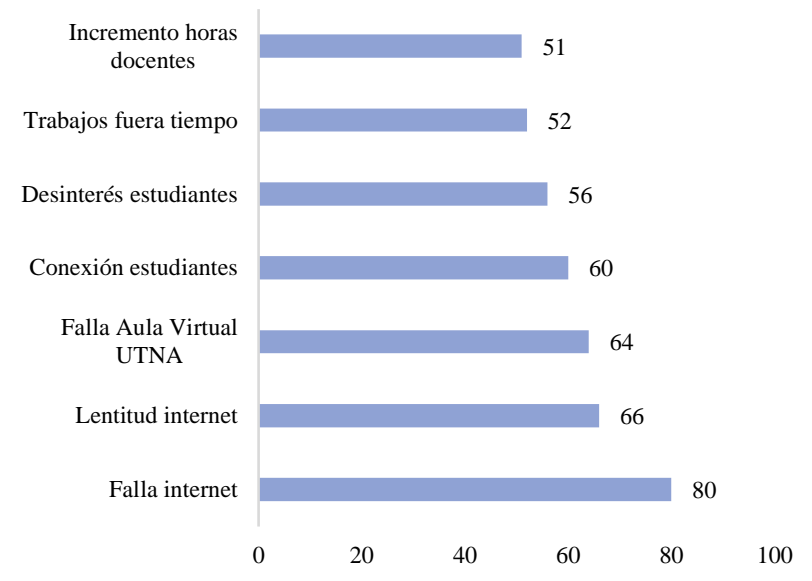

Gráfico 4 Principales dificultades enfrentadas por los docents

Acerca de las condiciones de apoyo institucional con las que han contado los profesores para la enseñanza remota. Se encontró que $82 \%$ de los docentes tomaron el Curso Entornos Virtuales y 73\% lo consideró útil para su práctica; además, la gran mayoría de los docentes tuvo oportunidad de compartir su experiencia con otros profesores (84\%) y más de la mitad ha recibido asesoría por parte de su dirección académica (55\%).

Considerando que la enseñanza remota se lleva a cabo a través de medios electrónicos, se exploró si los docentes contaban con equipo de cómputo y conexión a internet. Se encontró que seis de cada diez docentes tienen una computadora para uso exclusivo, mientras el $30 \%$ comparte su equipo, lo cual puede representar desafíos para la realización de las actividades de docencia, teniendo que articular sus horarios de trabajo con el equipo con el de las personas con quienes lo comparte. Por otro lado, únicamente $17 \%$ de los docentes dispone de una laptop institucional.

También se indagó sobre el acceso a servicio de internet y la disponibilidad de un espacio adecuado para el trabajo en casa. Casi la totalidad de los docentes cuenta con este servicio en sus hogares $(94 \%)$, pero solo poco más de la mitad reportó contar con un espacio de trabajo adecuado en casa (ver gráfico 5). 


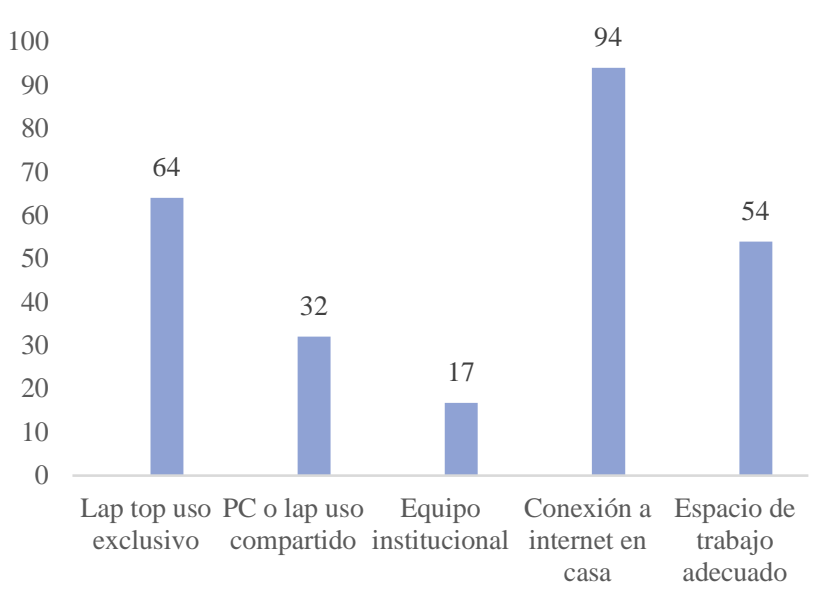

Gráfico 5 Condiciones para el trabajo en casa

En el cuestionario se preguntó por dificultades personales que enfrentaron los docentes durante el periodo de enseñanza remota, pues pueden convertirse en una fuente adicional de estrés. En el gráfico 6 se observa que los problemas que experimentaron un cuarto o más de los docentes fueron: problemas de salud mental (35\%), pérdidas económicas (26\%) y dificultades laborales (25\%).

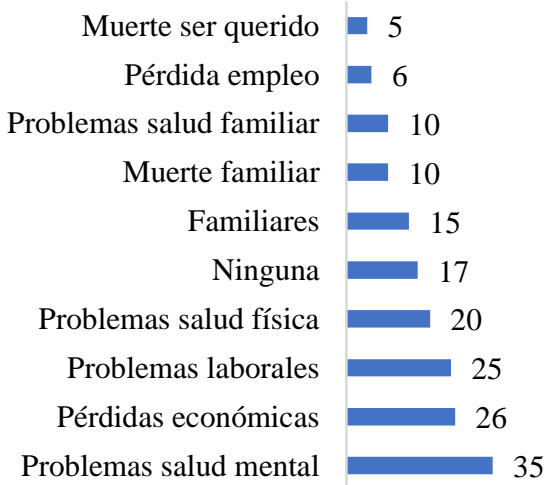

Gráfico 6 Dificultades personales enfrentadas durante la pandemia

Los hallazgos muestran la importancia de cuidar las condiciones en las cuales se instrumenta la educación remota a fin de poder estimar y valorar sus efectos.

\section{Conclusiones}

Los resultados de este estudio evidencian desafíos que han enfrentado los docentes durante la educación remota instrumentada en respuesta a la pandemia por COVID-10.
Un desafío ha sido la implementación remota de programas que fueron diseñados para educación presencial, pues de acuerdo con las respuestas de los docentes, perciben que han dedicado una mayor cantidad de tiempo a actividades de planificación y evaluación que lo que destinaban antes de la pandemia. Además, en el desarrollo de sus funciones remotas han tenido que emplear recursos y servicios personales, en caso de contar con ellos, como equipo de cómputo e internet.

Es probable que el tiempo que dedican los docentes a actividades como planificación y evaluación en la ERE se deba a que se han visto impulsados a desarrollar materiales educativos virtuales, sin contar con la expertise necesaria. En conjunto, esto se puede traducir en una fuente de estrés para los profesores, a la que se pueden agregar otras dificultades como falta o ineficiencia de conexión de internet, fallas en la plataforma virtual y falta de respuesta de algunos de los estudiantes.

Adicionalmente la realización de las funciones de docencia completamente desde casa ha representado condiciones diferenciadas de trabajo para los docentes. Algunos comparten el equipo de cómputo con sus familiares lo que puede significar una disminución del tiempo efectivo de trabajo a distancia o una prolongación del tiempo destinado a la preparación y evaluación de clases. Además, la mitad de los docentes no dispone de un espacio adecuado para el trabajo académico.

Los resultados de este estudio muestran que los docentes han enfrentado diferentes dificultades durante este periodo de confinamiento, como pérdidas económicas, de salud física o mental o pérdida de algún ser querido. Las pérdidas económicas pueden ser particularmente preocupantes para los docentes cuya contratación es de asignatura, pues realizan actividades laborales complementarias.

Como se señala en la literatura, el fenómeno observado en la institución coincide con la noción de enseñanza remota de emergencia. La prestación de servicios educativos en las condiciones actuales ha implicado decisiones temporales y apremiantes, y esto ha tenido implicaciones en las prácticas docentes y otras actividades de las instituciones educativas. 
En la institución estudiada, como en muchas otras instituciones de educación superior, la respuesta inmediata fue transitar la prestación de servicios educativos a través del uso de internet. Esta migración urgente es poco probable que haya venido acompañada de un adecuado diseño instruccional, que se considera como una condición para la efectividad de la educación en línea. Por otro lado, en el periodo de enseñanza remota los docentes no se han dedicado exclusivamente a actividades de docencia, sino que sus prácticas han comprendido también tutorías, actividades de apoyo a estadías, entre otras actividades académicas y administrativas. Los desafíos de la realización de estas actividades complementarias podrían ser estudiados en el futuro.

Las condiciones descritas muestran la importancia de proveer estrategias de apoyo a la docencia y de atención al bienestar integral de los docentes, pues se estima que el periodo de ERE podría prolongarse al menos hasta que se cuente con una vacuna que logre disminuir el efecto de la pandemia.

Se requieren estudios de más largo alcance para profundizar en el conocimiento de las prácticas docentes. En este sentido, en la institución se ha iniciado la observación de las aulas virtuales.

Por otra parte, será importante indagar sobre los aprendizajes que han tenido lugar en la comunidad de académicos y entre los estudiantes con los cambios introducidos con la educación remota en el contexto de emergencia sanitaria.

\section{Referencias}

Ally, M. (2008). Foundations of Educational Theory for Online Learning. En T. Anderson (Ed.), The theory and practice of online learning (2. ${ }^{\mathrm{a}}$ ed., pp. 15-44). AU Press. https://read.aupress.ca/read/the-theory-andpractice-of-online-learning/section/8949f7236904-454a-9704-6c241fb891ab\#ch01

Bozkurt, A., \& Sharma, R. C. (2020). Emergency remote teaching in a time of global crisis due to CoronaVirus pandemic. Asian Journal of Distance Education, 15(1), i-vi.
Casillas, J. C. S., \& Rodríguez, S. V. (2020). El docente universitario frente a las tensiones que le plantea la pandemia. Revista Latinoamericana de Estudios Educativos, 50(ESPECIAL), 89120 .

Diario Oficial de la Federación (16 de marzo de 2020). Acuerdo número 02/03/20 por el que se suspenden las clases en las escuelas de educación preescolar, primaria, secundaria, normal y demás para la formación de maestros de educación básica del Sistema Educativo Nacional, así como aquellas de los tipos medio superior y superior dependientes de la Secretaría de Educación Pública.

Galindo, D., García, L., Sánchez, R. G., Flores, P. G., Cerrito, P. C. H., Acosta, M. L., de la Luz, V. L., \& Arellano, C. I. M. (2020). Recomendaciones didácticas para adaptarse a la enseñanza remota de emergencia. Revista Digital Universitaria, 21(5).

Hodges, C., Moore, S., Lockee, B., Trust, T., \& Bond, A. (2020, marzo 27). The Difference Between Emergency Remote Teaching and Online Learning. EDUCASE Review. https://er.educause.edu/articles/2020/3/thedifference-between-emergency-remoteteaching-and-online-learning

Means, B., Bakia, M., \& Murphy, R. (2014). Learning Online: What Research Tells Us About Whether, When and How. Routledge.

Rugube, T., Mthethwa-Kunene, K. E., \& Maphosa, C. (2020). Promoting interactivity in online learning - towards the achievement of high-quality online learning outcomes. European Journal of Open Education and ELearning Studies, 5(2), 120-133. https://doi.org/10.46827/ejoe.v5i2.3381

Secretaría de Educación Pública (2020). Lineamientos de acción COVID-19 para instituciones públicas de educación superior. México:

SEP. http://www.anuies.mx/recursos/pdf/LINEAMIE NTOS_COVID-19_IES_SES_VFINAL.pdf

Stufflebeam, D. L., \& Zhang, G. (2017). The CIPP Evaluation Model: How to Evaluate for Improvement and Accountability. The Guilford Press. 
Sun, A., \& Chen, X. (2016). Online Education and Its Effective Practice: A Research Review. Journal of Information Technology Education: Research, 15, 157-190. https://doi.org/10.28945/3502

United Nations. (2020). Education during COVID-19 and beyond. Policy Brief. United Nations.

https://www.un.org/sites/un2.un.org/files/sg_po licy_brief_covid-

19_and_education_august_2020.pdf

Whittle, C., Tiwari, S., Yan, S., \& Williams, J. (2020). Emergency remote teaching environment: A conceptual framework for responsive online teaching in crises. Information and Learning Sciences, 121(5/6), 311-319. https://doi.org/10.1108/ILS-04-2020-0099

World Bank. (2020). The COVID-19 Crisis Response: Supporting tertiary education for continuity, adaptation, and innovation. World Bank Group Education. https://openknowledge.worldbank.org/bitstream /handle/10986/34571/The-COVID-19-CrisisResponse-Supporting-Tertiary-Education-forContinuity-Adaptation-and-

Innovation.pdf? sequence $=1$ \&isAllowed $=\mathrm{y}$. 